\title{
Nonlinear behavior of some hydrostatically stressed isotropic elastomeric foams
}

\author{
J. K. Dienes and J. C. Solem, Los Alamos, New Mexico \\ (Received June 15, 1998; revised October 30, 1998)
}

Summary. We calculate the stress-strain relation for elastomeric foam from an $a b$ initio theory, which shows that the "plateau" and "densification" regions should be described by a hyperbola. The theory seems to agree reasonably well with experiment.

\section{Introduction}

Dienes [1] has suggested that the principle of superposition of strain rates has much wider application than usually assumed, and that it can serve as a basis for formulating constitutive relations for highly nonlinear material behavior. In general, the relations involve tensors, but here we consider isotropic behavior, which requires only scalars. For hydrostatically stressed isotropic foam, the strain rate can be expressed as the sum of a term involving a changing stress and a term that represents the changing compliance due to compaction, so

$\dot{\varepsilon}=C \dot{\sigma}+\dot{C} \sigma$,

where $\varepsilon$ is the strain, $C$ is the compliance, $\sigma$ is the stress, and a dot denotes the rate of the underlying quantity.

In this paper we apply the principle of superposition of strain rates to obtain the nonlinear behavior of certain elastomeric foams. Section 2 reviews the theory of a spherical void. Though well known, the results are needed in the subsequent calculations combining the behavior of an ensemble of voids. Section 3 accounts for boundary conditions on a single void and Sect. 4 considers multiple voids. Section 5 demonstrates the validity of results in the limit of an infinite bulk modulus. In Sect. 6 we apply the method to the most frequently encountered case for elastomeric foams: finite bulk modulus, large compared to shear modulus. In this case we find the stress-strain curve to be a hyperbola. Then, in Sect. 7, we compute the effective shear modulus, and in Sect. 8 we show the results to be consistent with experimental data at large compressions for three types of foam. The transition region between linear behavior and complete buckling is very short, and not of particular interest here, but it can be shown using a nonlinear buckling theory that the transition is very quick, as observed in experiments. We conclude with a tentative explanation for why the results of an apparently naive theory agree so well with data in a highly nonlinear regime. 


\section{Behavior of a single void}

Consider a thin spherical shell of inner radius $r$ and thickness $\Delta r$. Equating the force normal to an equatorial section to the corresponding component of force resulting from radial stress leads to the result

$2 \pi r \Delta r \sigma_{\theta}=\Delta\left(\pi r^{2} \sigma_{r}\right) \simeq \pi r^{2} \Delta \sigma_{r}+2 \pi r \sigma_{r} \Delta r$,

where $\sigma_{r}$ is the radial stress and $\sigma_{\theta}$ is the tangential stress. Rearranging Eq. (2) and taking the limit, we have

$2\left(\sigma_{\theta}-\sigma_{r}\right)=r \frac{d \sigma_{r}}{d r}$

From Hooke's law and Poisson's principle in spherical symmetry, the radial strain is

$\varepsilon_{r}=\frac{\sigma_{r}}{E}-2 \nu \frac{\sigma_{\theta}}{E}=\frac{d u}{d r}$,

and the tangential strain is

$\varepsilon_{\theta}=\frac{\sigma_{\theta}}{E}-\nu \frac{\sigma_{r}+\sigma_{\theta}}{E}=\frac{u}{r}$,

where $E$ is Young's modulus, $\nu$ is Poisson's ratio, and $u$ is the radial displacement at the radius $r$. Thus,

$r=r(\bar{r}, t)$

is the current radius of a particle in the elastic material, initially at $\bar{r}$. So $r(\bar{r}, 0)=\bar{r}$ and the displacement is $u=r-\bar{r}$. (The bar is used throughout to denote the initial value of a variable.)

The solution to Eqs. (3), (4), and (5) is

$u=a r+\frac{b}{r^{2}}$.

It is readily shown that the mean stress, $\left(\sigma_{r}+2 \sigma_{\theta}\right) / 3$, is unaffected by the cavity, taking on the far-field value of the radial stress, $\sigma_{r}{ }^{(\infty)}$, everywhere in the material.

\section{Boundary conditions}

Equation (7) applies to any sphere of elastic material. Now we consider a sphere of very large outer radius with a concentric spherical cavity of radius $r_{0}$. We evaluate $a$ and $b$ from the boundary conditions. First, $\sigma_{r}$ must approach the constant $\sigma_{r}{ }^{(\infty)}$ as $r \rightarrow \infty$. Given the radial stress in the far field,

$a=\frac{(1-2 \nu) \sigma_{r}{ }^{(\infty)}}{E}=\frac{\sigma_{r}{ }^{(\infty)}}{3 k}$,

where $k=E / 3(1-2 \nu)$ is the bulk modulus. Second, the radial stress must vanish for $r=r_{0}$, which gives

$b=\frac{(1+\nu) r_{0}{ }^{3} \sigma_{r}{ }^{(\infty)}}{2 E}=\frac{r_{0}{ }^{3} \sigma_{r}{ }^{(\infty)}}{4 \mu}$, 
where $\mu=E / 2(1+\nu)$ is the shear modulus or Lamé's constant. Combining Eqs. (7), (8), and (9), we obtain the displacement

$u=\frac{(1-2 \nu) r \sigma_{r}(\infty)}{E}+\frac{(1+\nu) r_{0}{ }^{3} \sigma_{r}(\infty)}{2 E r^{2}}$.

The displacement is unaffected by the presence of a cavity for large $r$. This result, which follows from the displacement solution of the elastic equation, is consistent with the superposition principle, i.e., the continuum superposition principle is supported in the example of a spherical void: the overall strain is the sum of the continuum part and a defect part.

\section{Multiple voids}

To fix ideas, first note that the volumetric strain is independent of $r$, because combining Eqs. (4), (5), and (6), we have

$\varepsilon_{r}+2 \varepsilon_{\theta}=\frac{\sigma_{r}(\infty)}{k}$.

This local strain is different from the macroscopic strain, which will be discussed later. The deviatoric strain [2], $e_{i j}=\varepsilon_{i j}-\frac{1}{3} \varepsilon_{k k} \delta_{i j}$, depends only on the shear modulus,

$e_{r}=e_{11}=-\frac{\sigma^{(\infty)}}{2 \mu} \frac{r_{0}^{3}}{r^{3}}$

$e_{\theta}=e_{22}=\frac{\sigma^{(\infty)}}{4 \mu} \frac{r_{0}^{3}}{r^{3}}$

which can be easily derived from Eqs. (4), (5), and (10). Thus, the material adjacent to the void is not subject to any density change due to the presence of the void, although it displaces outward and changes shape.

From Eq. (7), the outward volume displacement for each void at $r$ is

$4 \pi u r^{2}=4 \pi a r^{3}+4 \pi b$.

Since $a=\sigma_{r}{ }^{(\infty)} / 3 k$ (see Eq. (8)), the first term is just the volume change due to homogeneous bulk behavior. But the second term involves the volume of the void, since from Eq. (9) there follows

$4 \pi b=\frac{\pi r_{0}^{3} \sigma_{r}(\infty)}{\mu}$.

Because the volume change due to a void is independent of the distance from the void, we can expect the effect of a number of voids to add.

Consider now the behavior of an ensemble of voids. Let $N$ be the number density of voids per unit mass in a porous solid (foam), a quantity that does not change with deformation. Then, $N \varrho$ is the number per unit volume, with $\varrho$ the density of the solid material. Since the first term of Eq. (14) is independent of the voids and the second term is independent of the radius, but proportional to the cavity volume, it is natural to write the volume displaced through the surface of a sphere $S$ with radius $\widetilde{r}$ as

$$
\Delta V=4 \pi u \tilde{r}^{2}=a \cdot 4 \pi r^{3}+b \cdot 4 \pi \frac{4}{3} \pi N \varrho \tilde{r}^{3},
$$


where $\frac{4}{3} \pi \varrho N \tilde{r}^{3}$ is the number of voids in $S$. The sphere is not symmetrical about each void, so the displacement is not exactly the same as if it were symmetric. But if $\tilde{r} \gg r_{0}$, it should matter little. The average volume strain for the ensemble is

$\frac{\Delta V}{V}=3 a \frac{r^{3}}{\tilde{r}^{3}}+4 \pi b N \varrho$.

Using Eqs. (8) and (9), we can rewrite Eq. (17) as

$\frac{\Delta V}{V}=\frac{\sigma_{r}{ }^{(\infty)}}{k} \frac{r^{3}}{\tilde{r}^{3}}+\frac{\pi r_{0}{ }^{3} \sigma_{r}{ }^{(\infty)} \varrho N}{\mu}=\frac{\sigma_{r}{ }^{(\infty)}}{k} \frac{r^{3}}{\tilde{r}^{3}}+\frac{3}{4} \frac{\sigma_{r}{ }^{(\infty)}}{\mu} f$,

where $f=\frac{4}{3} \pi N \varrho r_{0}{ }^{3}$ is the void fraction. To close the equations, we write an additional relation involving $\bar{f}$, where $\bar{f}=\frac{4}{3} \pi N \varrho \bar{r}_{0}^{3}$, the initial value of $f$. In fact, the change in the void fraction plus the change in the absence of voids is the change in volumetric $\operatorname{strain}^{1} \Delta V / V$,

$f-\bar{f}+\frac{\sigma_{r}(\infty)}{k}=\frac{\Delta V}{V}$.

Substituting Eq. (8) into Eq. (18), combining with Eq. (19) to eliminate $f$, and letting $\tilde{r} / r \rightarrow 1$, we obtain

$\frac{\Delta V}{V}=\frac{\sigma_{r}{ }^{(\infty)}}{k}+\frac{3 \sigma_{r}{ }^{(\infty)}}{4 \mu}\left(\bar{f}+\frac{\Delta V}{V}-\frac{\sigma_{r}{ }^{(\infty)}}{k}\right)$.

Changing signs in Eq. (20) so $\Delta V$ is positive under compression by making the substitutions $\sigma_{r}(\infty) \rightarrow-\sigma$ and $\frac{\Delta V}{V} \rightarrow-\varepsilon$, we have, on dropping the superscript,

$\varepsilon=\frac{\sigma}{k}+\frac{3 \sigma}{4 \mu}\left(\bar{f}-\varepsilon+\frac{\sigma}{k}\right)$.

Equation (21) represents a hyperbola with one asymptote parallel to the strain axis and the other asymptote at the slope of the bulk modulus $k$. The second asymptote intersects the strain axis at $\bar{f}$. Equation (21) is quadratic in $\sigma_{r}$, and the physically meaningful solution is

$\sigma=\frac{1}{2} k(\varepsilon-\bar{f})-\frac{2}{3} \mu-\frac{1}{6} \sqrt{[3 k(\varepsilon-\bar{f})-4 \mu]^{2}+48 k \mu \varepsilon}+c$,

where a constant of integration $c$ is added to account for the stress contribution at the transition to buckling, as will be discussed in a later section.

\section{Limit for very high stress}

In the limit of very large stress, all the cavities should collapse, and the stress-strain relation should be the same as for the bulk polymer, i.e., $d \sigma=k d \varepsilon$. Differentiating Eq. (22), we find

$\frac{d \sigma}{d \varepsilon}=\frac{1}{2} k\left\{1+\frac{3 k(\varepsilon-\bar{f})+4 \mu}{\sqrt{[3 k(\varepsilon-\bar{f})-4 \mu]^{2}+48 k \mu \varepsilon}}\right\}$,

\footnotetext{
1 In other words, the presence of the voids does not affect the compression of the adjacent material.
} 
and taking the appropriate limit

$\lim _{k \rightarrow \infty} \frac{d \sigma}{d \varepsilon}=k$

which is exactly what we would expect.

\section{Approximation for large bulk modulus}

In general we expect the shear modulus to be small compared to the bulk modulus for elastomeric materials, which typically have a Poisson ratio near $1 / 2$. This allows a useful approximation, which eliminates the need for knowledge of the bulk modulus. If we expand Eq. (22) as a Taylor series in $k^{-1}$, we obtain

$\sigma=\frac{2}{3} \mu\left(\frac{\varepsilon+\bar{f}}{\bar{f}-\varepsilon}-1\right)+c=\frac{4}{3} \frac{\mu \varepsilon}{\bar{f}-\varepsilon}+c$,

which is the familiar hyperbolic function with orthogonal asymptotes.

\section{Effective shear modulus}

Nemat-Nasser and Hori [1] have shown that the effective shear modulus of an elastic solid with spherical voids, using their differential scheme, is given by

$\frac{\partial \bar{\mu}}{\partial \bar{f}}=\frac{\bar{\mu}}{(1-\bar{f})\left(1-s_{2}\right)}$,

where $\vec{\mu}$ is the effective shear modulus and

$s_{2}=\frac{2}{15} \frac{4-5 \nu}{1-\nu}$.

Integrating Eq. (26) we have

$\bar{\mu}=\mu_{s}(1-\bar{f})^{\alpha}$,

where $\mu_{s}$ is the shear modulus of the solid (matrix) material and

$\alpha=\frac{15(1-\nu)}{7-5 \nu}$.

Substituting Eq. (29) into Eq. (25), we have

$\sigma=\frac{4}{3} \frac{\mu_{s} \varepsilon(1-\bar{f})^{\alpha}}{\bar{f}-\varepsilon}+c$

\section{Comparison with experiment}

Figure la shows a family of experimental stress-strain curves for open-cell polyethylene foam [4], taken at low strain rate $\left(\dot{\varepsilon} \sim 10^{-2} \mathrm{~s}^{-1}\right)$, so the material is essentially in equilibrium. The densities of the uncompressed foams are $138,120,69.2$, and $29.4 \mathrm{~kg} \cdot \mathrm{m}^{-3}$. Figure $1 \mathrm{~b}$ shows a 

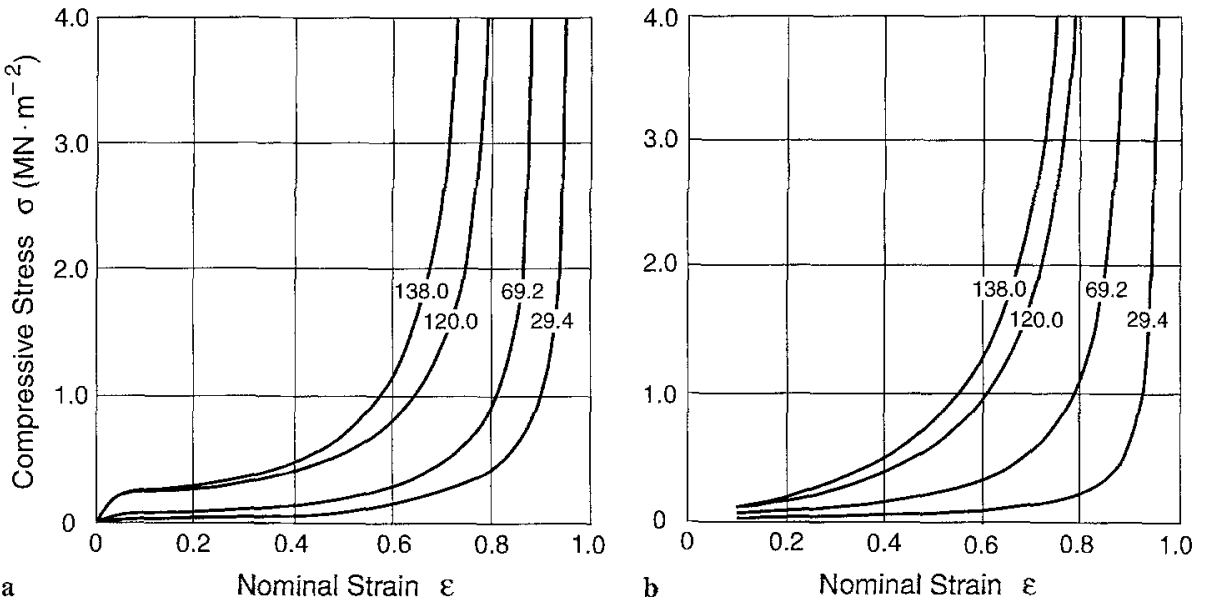

Fig. 1. Polyethylene foam: a Experimental stress-strain curves with uncompressed densities in $\mathrm{kg} \cdot \mathrm{m}^{-3} ; \mathbf{b}$ theoretical values for the same densities
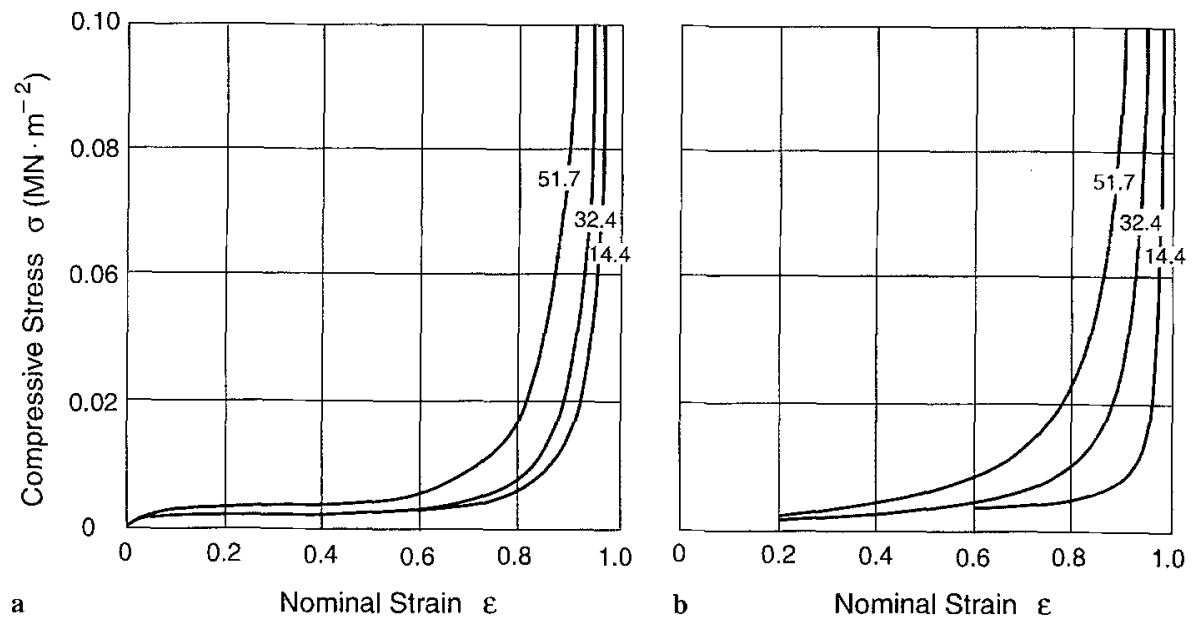

Fig. 2. Polyurethane foam: a Experimental stress-strain curves with uncompressed densities in $\mathrm{kg} \cdot \mathrm{m}^{-3}$; b theoretical values for the same densities

family of theoretical stress-strain curves for the same densities as calculated from Eq. (30), assuming a solid polymer density of $1 \mathrm{Mg} \cdot \mathrm{m}^{-3}$ and solid shear modulus [5] of $\mu_{s}=10 \mathrm{MN} \cdot \mathrm{m}^{-2}$ and a Poisson's ratio of $\nu=1 / 2$. The curves are cut off below a strain of 0.2 . In the low strain region, there is a linear response of stress to strain until buckling occurs, at which point the curve rapidly turns down to the plateau region. We use the term buckling constant for the stress at the onset of the plateau region. This is the same constant $c$ as first mentioned near the end of Sect. 4. In Fig. 1, the buckling constants for the curves are all left at zero to emphasize the semiquantitative agreement between the theoretical and experimental results. The buckling constant will increase with density, causing the curves in Fig. $1 \mathrm{~b}$ to more closely agree with the curves in Fig. 1a. The low-stress region is not applicable to the theory presented here, and an analysis of buckling will be addressed in a future paper.

Figure 2a shows a family of experimental stress-strain curves for open-cell polyurethane foam, taken at a low strain rate $\left(\dot{\varepsilon} \sim 10^{-3} \mathrm{~s}^{-1}\right.$ ), so the material is essentially in equilibrium. The densities of the uncompressed foams are $51.7,32.4$, and $14.1 \mathrm{~kg} \cdot \mathrm{m}^{-3}$. Figure $2 \mathrm{~b}$ shows a 

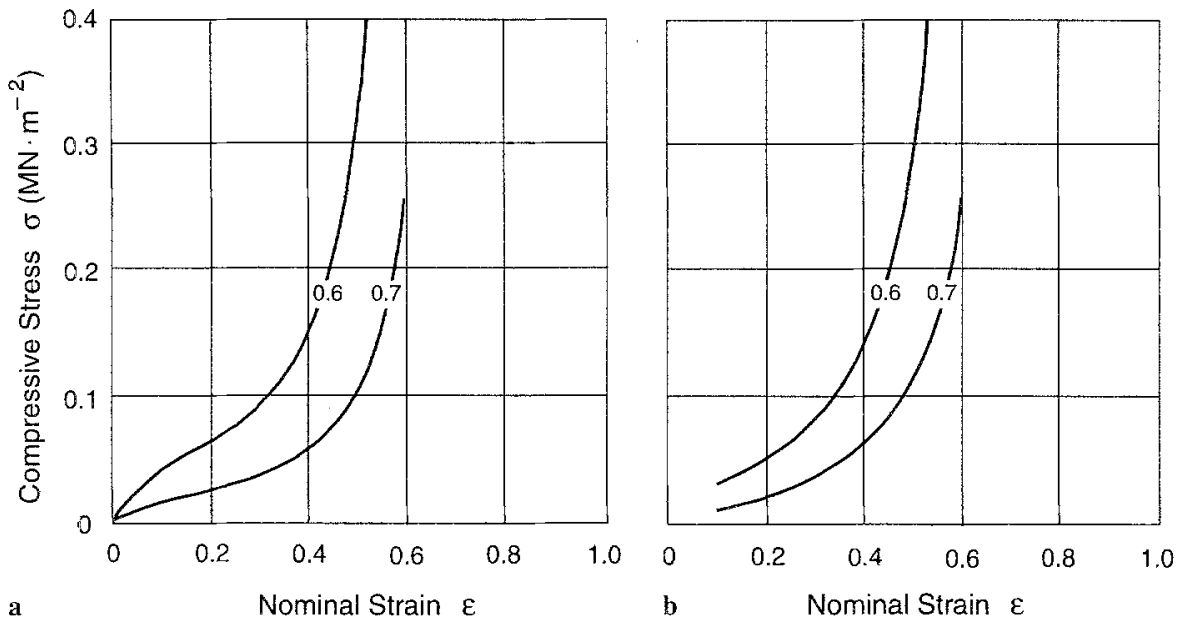

Fig. 3. Silicone foam: a Experimental stress-strain curves with uncompressed porosities; $\mathbf{b}$ theoretical values for the same porosities

family of theoretical stress-strain curves for the same densities as calculated from Eq. (30), assuming a solid polymer density of $1.2 \mathrm{Mg} \cdot \mathrm{m}^{-3}$ and $\mu_{s}=0.8 \mathrm{MN} \cdot \mathrm{m}^{-2}$. The curves are cut off below a strain of 0.2 as that is the region where buckling dominates. The buckling constants for the curves are $0.002,0.002$, and $0.004 \mathrm{MN} \cdot \mathrm{m}^{-2}$, respectively.

Figure 3a shows a pair of experimental stress-strain curves for open-cell silicone foam [6], also taken at low strain rate. The thickness of both samples was about $1 \mathrm{~mm}$. The porosities $\bar{f}$ are 0.6 and 0.7 Figure $3 \mathrm{~b}$ shows a pair of theoretical stress-strain curves as calculated from Eq. (30), assuming $\mu_{s}=0.27 \mathrm{MN} \cdot \mathrm{m}^{-2}$. The curves are cut off below a strain of 0.2 as that is the region where buckling dominates. The buckling constants for the curves are 0.02 and $0.005 \mathrm{MN} \cdot \mathrm{m}^{-2}$, respectively.

\section{Conclusion and comment}

On the basis of a very simple $a b$ initio calculation, we have shown that the nonlinear region of the stress-strain relation for elastomeric foams should be approximated by a hyperbola. We recognize that the problem is far more complex than the simplified picture we have presented. But we are gratified by the general agreement with the behavior of some polyethylene, polyurethane, and silicone foams, based only on the knowledge of the porosity and shear modulus of the bulk polymer.

The success of our simple calculation can be partially explained by referring to some comments by Kachanov, although, strictly speaking, they apply only to linear media. First, if his method of superposition [7] were applied to find the interactions within an ensemble of spherical holes, it would hinge on finding the mean stress at a cavity location (without the void) due to each of its cavity neighbors. This mean stress is uniform, however, and unaffected by the presence of a hole. Thus his superposition method, which is very similar to those described by a number of authors cited by Kachanov, would lead to the conclusion that the interaction has no effect, at least in that approximation. In a similar vein, Kachanov notes that competing effects of stress shielding and stress amplification balance each other - at least in the cases investigated [8]. 


\section{References}

[1] Dienes, J. K.: A unified theory of flow, hot spots and fragmentation with an application to explosive sensitivity. In: High pressure compression of solids II, 1 (Davidson, D. Grady, D., and Shahinpoor, M., eds.), pp. 372-380. Berlin Heidelberg New York Tokyo: Springer 1996.

[2] Mura, T.: Micromechanics of defects in solids, 2nd rev. ed., p. 182. Dordrecht Boston London: Kluwer 1991.

[3] Nemat-Nasser, S., Hori, M.: Elastic solids with microdefects. In: Micromechanics and inhomogeneity (Weng, G. J., Taya, M., Abé, H., eds.), p. 319. Berlin Heidelberg New York Tokyo: Springer 1990.

[4] Gibson, L. J., Ashby, M. F.: Cellular solids, 2nd ed., p. 180. Cambridge New York Melbourne: Cambridge University Press 1997.

[5] Ward, I. M.: Low density polyethylene 2. In: Mechanical properties of solid polymers, 2nd ed., p. 279. Chichester: Wiley 1983.

[6] Hemphill, D., Sandoval, C.: Personal communication (1997).

[7] Kachanov, M.: Elastic solids with many cracks and related problems. In: Advances in applied mechanics, vol. 30. (Hutchison, J. W., Wu, T. Y., eds.), Sect. IIIB. Boston: Academic Press 1994.

[8] Kachanov, M.: Elastic solids with many cracks and related problems. In: Advances in applied mechanics, vol. 30 (Hutchinson, J. W., Wu, T. Y., eds.), Sect. VIIIC. Boston: Academic Press 1994.

Authors' address: J. K. Dienes and J. C. Solem, Theoretical Division, Los Alamos National Laboratory, Los Alamos, New Mexico 87545, USA 